\title{
A theoretical study on the Supercontinuum Generation in a Novel Suspended Liquid Core Photonic Crystal Fiber
}

\author{
Sharafali.A ${ }^{1}$, K.Nithyanandan ${ }^{2,3 *}$ \\ ${ }^{1}$ Department of Physics, Pondicherry University, \\ Puducherry 605 014, India. \\ ${ }^{2}$ Institute of Photonics and Electronics of the CAS, \\ v.v.i., Chabersk 57, 18251 Prague, Czech Republic. \\ ${ }^{3}$ Optoelectronics Research Centre, \\ University of Southampton, Highfield, \\ Southampton SO17 1BJ, UK. \\ *Email: nithi.physics@gmail.com
}

\begin{abstract}
We theoretically propose a novel liquid filled suspended core photonic crystal fiber as a new class of micro-structure optical fiber for ultra broad supercontinuum generation. We emphasize the advantage of liquid infiltration in enhancing the fiber nonlinearity. To further enhance the nonlinearity of the liquid infiltrated fibers, we introduce a suspended liquid core photonic crystal fiber which significantly elevates the fiber nonlinearity through reduced effective area. A comparative study on the continuum generated in conventional microstructured optical fiber (without suspension effect) and the suspended core microstructured optical fiber is performed. A broad continuum is numerically demonstrated through the suspended core fiber, which is substantially broader than the fiber without suspension effect. Thus, we propose a new means to enhance the nonlinearity beyond the intrinsic material dependence. The underlined suspended liquid core photonic crystal fiber can be a new class of fibers for next generation of broadband laser sources.
\end{abstract}

\section{INTRODUCTION}

Rapid developments in micro structured optical fibers (MOFs) driven by novel engineering techniques continue to push the limits of light based technology and its applications. This major advancement in MOFs is essentially due to its high design flexibility in maneuvering the fiber characteristics by simply changing the fiber geometry [1]. Based on the geometry and the principle of operation, the MOFs are primarily classified into solid core photonic crystal fibers (PCFs) and hollow core photonic crystal fibers (HC-PCFs). Former usually consists of a set of cylindrical glass capillaries arranged in a regular lattice with a central defect, while the latter is the MOFs with a hollow core for band gap guidance [24]. MOFs also shows exceptional optical properties like high nonlinearity, high birefringence, endlessly single mode, and large mode area etc. thus makes it naturally attractive for a vast verity of applications like pulse compression, supercontinuum generation [5-7] etc.

There are several techniques have been proposed over the years to enhance the linear and nonlinear properties of MOFs by varying the geometrical parameters with the prearranged form [8-19]. One of the possible means of enhancing the nonlinearity associated with MOF structure is through non-silica material based technology [20]. For instance, liquid such as index oils [21, 22], polymers [23], metals [24] etc., have been used to infiltrate into the air hole to form non-silica based MOFs. These kind of MOFs are useful for different applications like switching, communication and optical sensing [21-29] etc. Due to the rapid development of material research and the advancement of fiber fabrication technology, there are many new fiber designs continue to reach the scientific community as well as the commercial market.

The present work in this paper is one such motivation, where we propose a novel nonlinear liquid based suspended core photonic crystal fiber as a new class of fibers for potential nonlinear applications. The research in liquid core MOFs (liquid core photonic crystal fiber (LCPCF), where the hollow core of the HC-PCF is infiltrated with appropriate nonlinear liquids) is known for a decade, and the quest for more efficient and hybrid materials for various applications continues to thrive research in fiber optics technology [27]. MOFs filled with liquids was used to produce very low chromatic dispersion by varying the size of air hole and low confinement loss by varying the number of air-hole layers in the cladding as reported in Refs. [28, 29]. LCPCFs have also been considered effectively as a tool for temperature sensing, fibered photon-pair generation, all optical wavelength conversion [30-32] etc. Recently, Ghosh et al., realized a novel PCF structure known as suspension core photonic crystal fiber (S-PCF) to enhance the nonlinearity by maneuvering the air hole geometry [33]. Thus inspired by the rapid technological advancement in the fiber fabrication process, we propose to merge the growing non-silica technology with the state-of-the-art fiber fabrication technique to realize a novel fiber structure known as suspended LCPCF (S-LCPCF). Through an extensive numerical simulation, we show that the proposed S-LCPCF exhibit enhanced nonlinearity as a result of liquid infiltration and core suspension. These kind of fibers can be of potential interest in various nonlinear applications.

Among the various nonlinear applications, one of the most advanced frontiers in nonlinear optics is the generation of intense ultra-broadband spectrum known as supercontinuum generation (SCG) [34-37]. SCG is a complex nonlinear phenomenon featuring dramatic spectral broadening of intense light pulses passing through a nonlinear media. The invention of MOFs also made much attraction towards the SCG as it can be used as an effective optical broadband source [7, 38-40]. SCG in optical fibers became an interesting area of research recently because of its potential applications in generation of ultra short pulses, frequency metrology, optical coherence tomography (OCT) systems [41-43]etc. For example, superluminescent diodes (SLDs) and amplified 
TABLE I: List of Acronyms and their description

\begin{tabular}{|c|c|}
\hline Acronyms & Description \\
\hline MOF & Micro structured optical fiber \\
\hline $\mathrm{PCF}$ & Photonic crystal fiber (solid core) \\
\hline $\mathrm{HC}-\mathrm{PCF}$ & Hollow core photonic crystal fiber \\
\hline $\mathrm{LCPCF}$ & Liquid core photonic crystal fiber \\
\hline $\mathrm{S}-\mathrm{PCF}$ & Suspended solid core photonic crystal fiber \\
\hline Silica PCF & Silica based solid core photonic crystal fiber \\
\hline $\mathrm{CCl}_{4} \mathrm{LCPCF}$ & $\mathrm{CCl}_{4}$ infiltrated liquid core photonic crystal fiber \\
\hline S-LCPCF & Suspended liquid core photonic crystal fiber \\
\hline S-Silica PCF & Silica based suspended core photonic crystal fiber \\
\hline $\mathrm{S}-\mathrm{CCl}_{4} \mathrm{LCPCF}$ & $\mathrm{CCl}_{4}$ infiltrated suspended liquid core photonic crystal fiber \\
\hline
\end{tabular}

spontaneous emission sources are the usual sources of OCT technique. With these sources the operations are restricted to certain limited bandwidth and wavelength range. These OCTs with 10-15 $\mu \mathrm{m}$ longitudinal resolutions are replaced with PCF based supercontinuum sources [44]. Supercontinuum can be generated by using PCF with high power picosecond or femtosecond laser pulses. Various research groups working in SCG has analyzed different mechanisms responsible for spectral broadening both theoretically as well as experimentally. Typically, the SCG is achieved by two popular mechanisms namely soliton fission and modulation instability (MI) [45]. Based on the input condition such as pulse width, peak power, either soliton fission or MI dominates the generating mechanism. For instance, with fixed input peak power, the soliton fission will be dominant in SCG process in fiber if the input pulse width is shorter, while MI take control of the SCG process for longer pulses [46].

Different approaches are made to obtain broad continuum with low input power in short length of fibers. As the material science engineering grows exponentially over the last decade, many novel nonlinear materials were developed, which rejuvenate the non-silica based fiber technology. Observation of different nonlinear phenomena in a hollow core fiber filled with nonlinear liquid was the very first successful attempt for nonlinearity enhancement with LCPCFs [47]. Recently, Zhang et.al., generated a very broad continuum with a hollow core fiber infiltrated with $\mathrm{CS}_{2}$ liquid [48]. Unlike silica, nonlinear liquids shows high nonlinearity and hence capable of producing a broad spectrum in a very short length of fiber at relatively less input power [49, 50]. Thus, inspired by the recent innovation in the fiber optic technology, we propose in this paper a highly nonlinear S-LCPCF as a potential fiber architecture for nonlinear applications with a particular emphasize on SCG.

The organization of the paper is as follows: Following a detailed introduction, Sec. II describes the modeling of the proposed PCF and its characteristics. Sec. III explains theoretical modeling and simulation results of SCG process. Sec. IV concludes the paper with a brief summary of results.

\section{DESIGNING THE SUSPENDED CORE PCF}

As the MOFs enable a delicate control over the fiber parameters like dispersion and nonlinearity, a variety of MOF structures have been proposed to achieve ultrashort pulses and broadband sources through techniques like pulse compression and SCG. Such fibers are typically scaled by two characteristic parameters namely, pitch (distance between the neighboring air holes represented by $\Lambda$ ) and the diameter of the cladding air holes $(d)$ as shown in Fig. (1a). During the fiber fabrication process, these parameters are delicately controlled by managing the drawing speed of the fiber, furnace temperature etc., in accordance with the required prearranged structure. However, for the present theoretical investigation we used the commercial software COMSOL 5.2a for fiber modeling and the mode analysis are performed by finite element method (FEM). To remove the back scattering at the boundaries of the simulation area, a circular shaped phase matched layer is imposed which has been the most efficient absorbing boundary condition [51]. In addition to the conventional route of changing the fiber parameters by varying the geometry, there exist alternate means by using non-silica material based core. Our presentation on the design of the S-LCPCF is two folds. Firstly, we review the concept of LCPCF by incorporating carbon tetrachloride $\left(\mathrm{CCl}_{4}\right)$ in the core of the $\mathrm{HC}-\mathrm{PCF}$. The choice of $\mathrm{CCl}_{4}$ in the present investigation is attributed to its high nonlinear refractive index $\left(\mathrm{n}_{2}\right)$ with almost same linear refractive index to that of silica. This equality in refractive index keep the dispersion value of all the proposed MOFs in almost same order and hence one can adopt large dimensions of fiber geometry with a very small shift in dispersion. Another advantage of using $\mathrm{CCl}_{4}$ is attributed to the fact that the nonlinear refractive index of $\mathrm{CCl}_{4}$ is significantly high compared to that of silica, and hence liquid infiltrated HC-PCFs became an active area of research in nonlinear optical community. The linear refractive index of $\mathrm{CCl}_{4}$ and silica are calculated by using the following formulae $[52,53]$

$$
n_{C C l_{4}}=\left(1+\frac{1.09215 \times \lambda^{2}}{\lambda^{2}-0.01187}\right)^{\frac{1}{2}}
$$

$$
n_{\text {silica }}=\frac{\left(0.788404+23.5835 \times 10^{-6} T\right) \times \lambda^{2}}{\lambda^{2}-\left(0.0110199+0.584758 \times 10^{-6} T\right)}+
$$




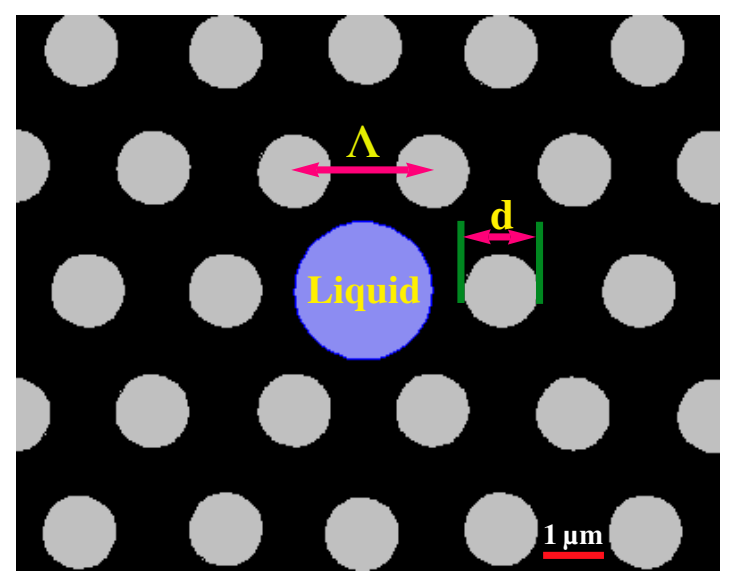

(a)

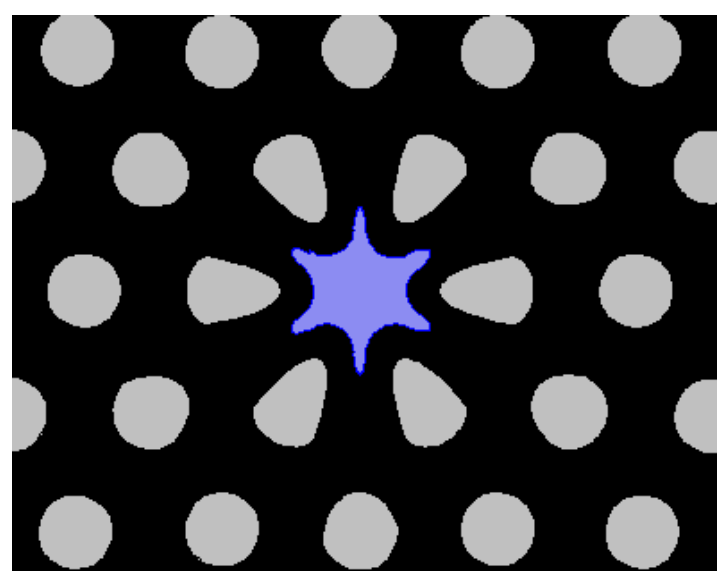

(b)

FIG. 1: (Color online) Cross sectional image of the proposed (a) liquid core PCF (LCPCF) and (b) suspended LCPCF (S-LCPCF).

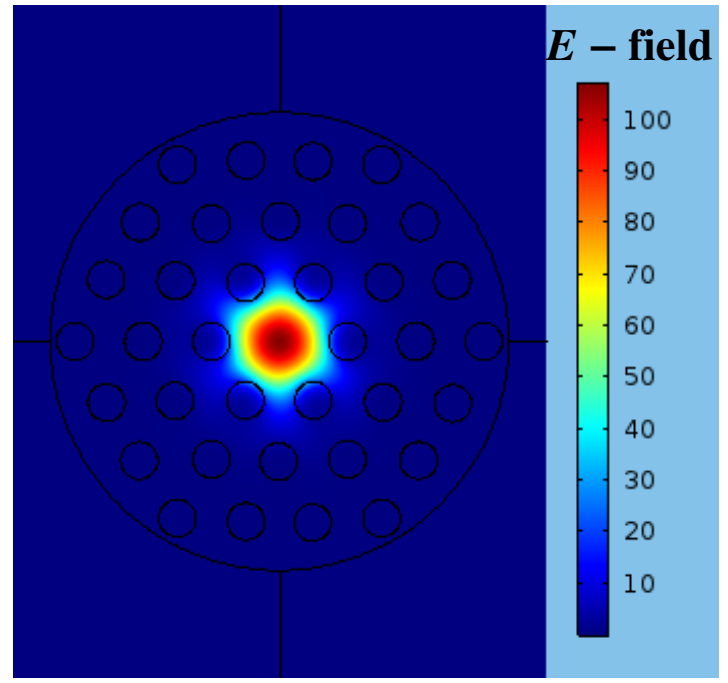

(a)

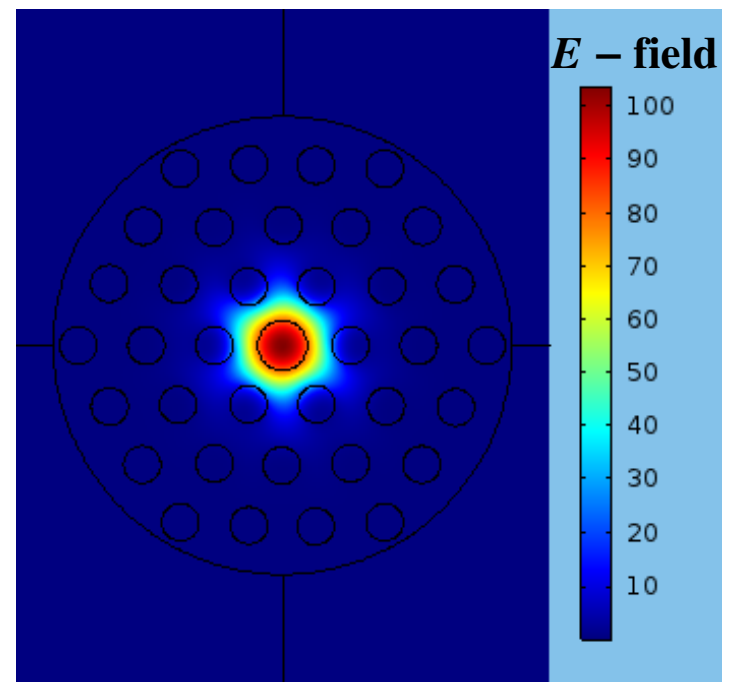

(c)

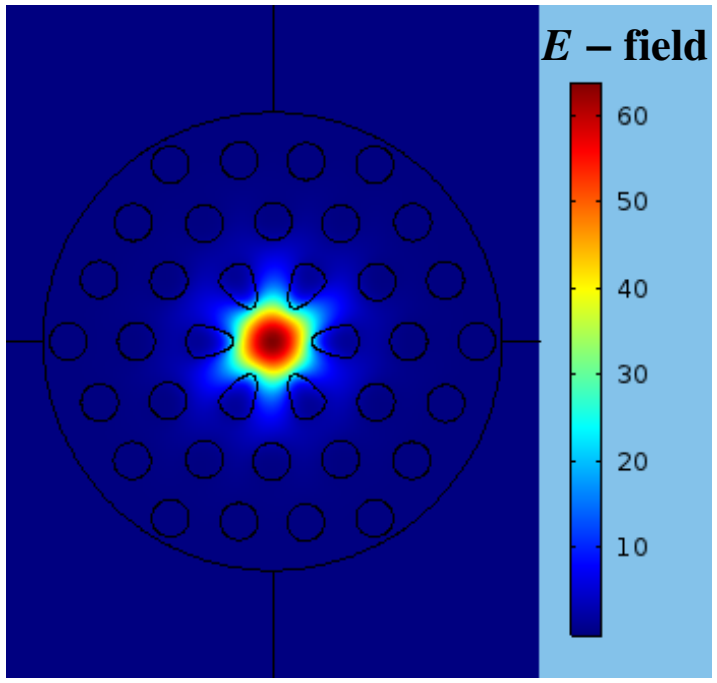

(b)

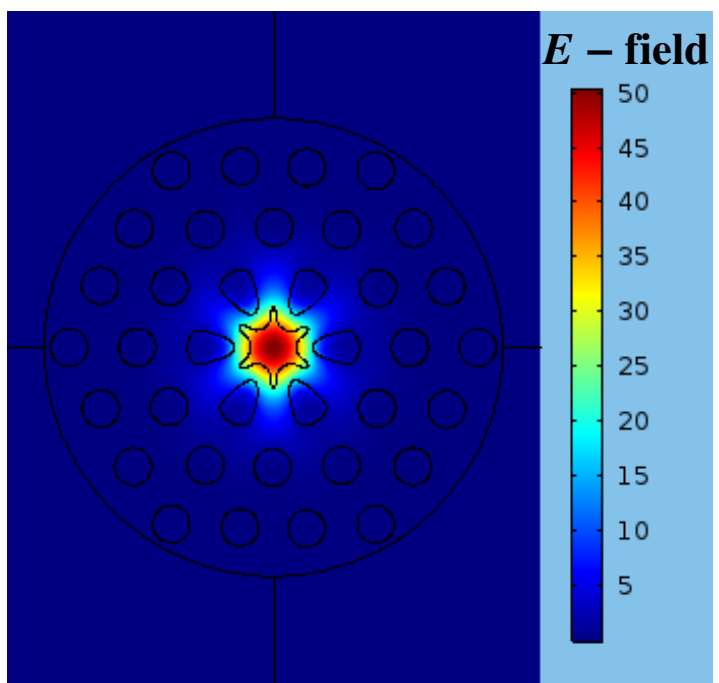

(d)

FIG. 2: (Color online) Fundamental mode distribution in (a) silica PCF, (b) S-Silica PCF, (c) $\mathrm{CCl}_{4} \mathrm{LCPCF}$ and (d) $\mathrm{S}-\mathrm{CCl}_{4} \mathrm{LCPCF}$.

$1.31552+6090754 \times 10^{-6} T+$ $\frac{\left(\left(0.91316+0.548368 \times 10^{-6} T\right) \times \lambda^{2}\right)}{\lambda^{2}-100}$.
Following a short review on LCPCF, in what follows, we briefly discuss the technique of core suspension as a potential new route to further enhance the nonlinearity of the LCPCF. A cross sectional view of the proposed 


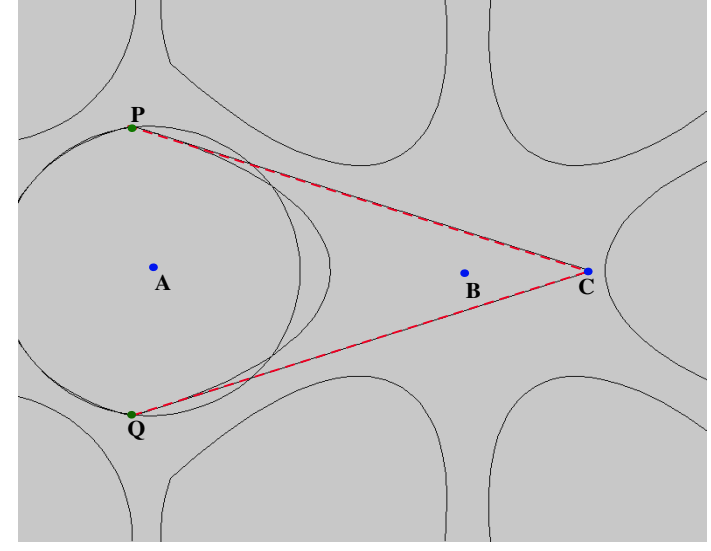

FIG. 3: (Color online) Schematic diagram of elongated primary air holes formed by Bèzier curve.

S-LCPCF is shown in Fig. (1b). The suspended core fiber can be manufactured by adjusting the fiber drawing parameters such as furnace temperature, feed rate, draw speed and differential pressure [33] etc. In the proposed model, the suspended core has a practically feasible structure designed by realizing in a hexagonal lattice MOF, where the six air-holes adjacent to the core are deformed into a conical shape towards the core as shown in Fig. (1b). This way of suspending the core can reduce the effective area and thereby substantially increases the nonlinearity of the fiber. This can be easily concluded from Fig. (2) where the effective mode area is substantially reduced as a result of suspension in the core region of the silica based S-PCF (S-silica PCF) and $\mathbf{C C l}_{4}$ core S-LCPCF. Also, the nonlinearity can be further increased with high suspension factor. For the simulations, we have used Bèzier polynomial [54], defined by Eq. (3), to model all the suspended core MOFs in this work. Where $\mathrm{P}, \mathrm{C}$ and $\mathrm{Q}$ are the three points given in Fig. (3) which defines the Bèzier curve. In Fig. (3) A and $\mathrm{B}$ are the center of first layer air hole and center of $\mathrm{MOF}$ respectively, which defines the suspension factor given as $\mathrm{SF}=\mathrm{AC} / \mathrm{AB}$. However, it should be noted that $\mathrm{SF}$ cannot be increased more than a critical value called $S F_{\max }$, which is governed by the geometrical parameters of the fibers $d$ and $\Lambda$. In this study, we have deformed the first layer of air hole with suspension effect having $\mathrm{SF}=1.27$. The minimum possible $\mathrm{SF}$ for this parametric ratio is $\mathrm{d} / \Lambda=0.545$ ( with $\mathbf{d}=\mathbf{1 . 2} \Lambda=\mathbf{2 . 2}$ ) and the maximum $\mathrm{SF}$ value is $2.5-(\mathrm{d} / \Lambda)=1.955$. Therefore, our choice of the $\mathrm{SF}$ value in the present context is practically feasible as it falls within the allowed limit as outlined in Ref. [33].

$$
B(x)=\left(1-x^{2}\right) P+2(1-x) x C+x^{2} Q, \quad x \in[0,1]
$$

As the change in refractive index with wavelength causes dispersion, the dispersion as a function of wavelength can be evaluated by using Eq. (4). Where $n_{\text {eff }}$ is the effective refractive index, $\lambda$ represents wavelength and $c$ is the velocity of light. We have used Eq. (5) to calculate the nonlinearity coefficient at different wavelength. Where, $A_{\text {eff }}$ represent the effective core area of the MOF designed, and $n_{2}$ is the nonlinear refractive index which can take values $3.5 \times 10^{-20}$, and $1.5 \times 10^{-19} \mathrm{~m}^{2} / \mathrm{W}$ respectively for silica, and $\mathrm{CCl}_{4}[49,50,55,56]$.

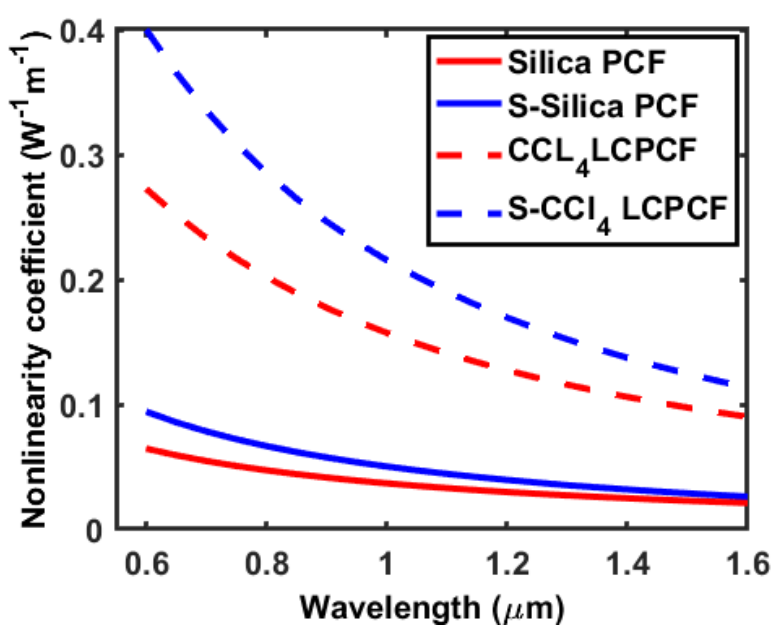

(a)

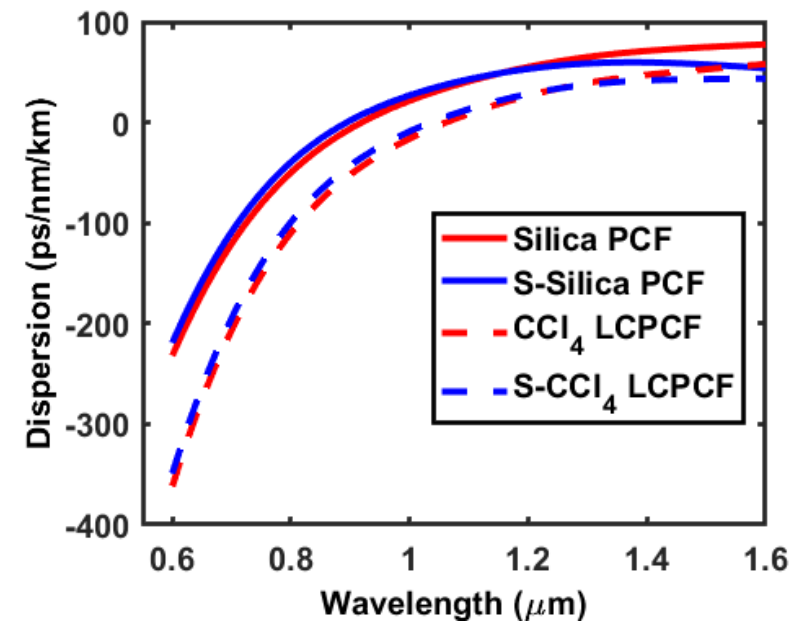

(b)

FIG. 4: (Color online) Variation of (a) coefficient of nonlinearity and (b) dispersion parameter with wavelength in different MOFs proposed with and without suspension.

TABLE II: Fiber parameters of all different MOFs design considered at pump wavelength $1.55 \mu \mathrm{m}$.

\begin{tabular}{|c|l|l|}
\hline \multicolumn{3}{|c|}{ MOF without suspension effect } \\
\hline Parameters & Silica PCF & CC $l_{4}$ LCPCF \\
\hline$\beta_{2}\left(\mathrm{ps}^{2} / \mathrm{m}\right)$ & -0.0974 & -0.0706 \\
\hline$\beta_{3}\left(\mathrm{ps}^{3} / \mathrm{m}\right)$ & $3.084 \times 10^{-5}$ & $3.041 \times 10^{-5}$ \\
\hline$\beta_{4}\left(\mathrm{ps}^{4} / \mathrm{m}\right)$ & $-6.272 \times 10^{-9}$ & $-1.041 \times 10^{-8}$ \\
\hline$\gamma\left(\mathrm{W}^{-1} \mathrm{~m}^{-1}\right)$ & 0.0219 & 0.0937 \\
\hline \multicolumn{3}{|c|}{ MOF with suspension effect } \\
\hline Parameters & S-Silica PCF & S-CCl $l_{4} \mathbf{L C P C F}$ \\
\hline$\beta_{2}\left(\mathrm{ps}^{2} / \mathrm{m}\right)$ & -0.0712 & -0.0552 \\
\hline$\beta_{3}\left(\mathrm{ps}^{3} / \mathrm{m}\right)$ & $8.283 \times 10^{-6}$ & $1.516 \times 10^{-5}$ \\
\hline$\beta_{4}\left(\mathrm{ps}^{4} / \mathrm{m}\right)$ & $-1.949 \times 10^{-10}$ & $-2.801 \times 10^{-8}$ \\
\hline$\gamma\left(\mathrm{W}^{-1} \mathrm{~m}^{-1}\right)$ & 0.0274 & 0.119 \\
\hline
\end{tabular}




$$
\begin{aligned}
D(\lambda) & =-\frac{\lambda}{c} \frac{d^{2} n_{e f f}}{d \lambda^{2}} \\
\gamma & =\frac{2 \pi n_{2}}{\lambda A_{e f f}}
\end{aligned}
$$

To demonstrate the effect of liquid infiltration in the fiber parameters, we have calculated the dispersion and nonlinearity coefficients of the silica $\mathrm{PCF}$ and $\mathrm{CCl}_{4}$ infiltrated $\mathrm{LCPCF}\left(\mathrm{CCl}_{4} \mathrm{LCPCF}\right)$ without any suspension effect. It has been observed that the nonlinearity coefficients corresponding to LCPCF is very high as depicted in Fig. (4a); this attribution is due to the high nonlinear refractive index of $\mathrm{CCl}_{4}$, which is consistent with the earlier reports on LCPCF [48]. Nonlinearity coefficient of 0.0219 and $0.0937 W^{-1} \mathrm{~m}^{-1}$ are evaluated for silica PCF and $\mathrm{CCl}_{4}$ LCPCF without suspension effect, at pump wavelength $\lambda_{p}=1550 \mathrm{~nm}$. A significant enhancement of nonlinearity $\approx 5$ times more than silica $\mathrm{PCF}$ is noticed, which substantiate convincingly that $\mathrm{CCl}_{4} \mathrm{LCPCF}$ is better than the silica PCF. The dispersion characteristics of each MOFs considered are studied up to fourth order for all combinations and the corresponding dispersion curves are depicted in Fig. (4b). A complete list of fiber parameters like dispersion and nonlinearity coefficients for all different choice of fibers are listed in Tab.II.

We now discuss the effect of core suspension in the LCPCF and compare with the above results to emphasize the effect of suspension. Firstly, the nonlinearity coefficients corresponding to S-silica PCF are evaluated and depicted in Fig. (4a). It has been observed that, irrespective of fiber materials the suspension effect enhance the nonlinearity by reducing the effective area. The dispersion characteristics of the silica with and without-suspension have also been evaluated and plotted in Fig. (4b). It is evident that there is only a slight variation in the dispersion curve, while the global variation remains unchanged by the suspension effect. This is particularly attractive for nonlinear application where one can effectively manipulate the nonlinearity without appreciable effect on the dispersion characteristics of that particular fiber type.

In similar lines, we study the effect of suspension in LCPCF. The variation of nonlinearity and dispersion with wavelength in the $\mathrm{CCl}_{4}$ infiltrated S-LCPCF (S$\mathrm{CCl}_{4} \mathrm{LCPCF}$ ) is shown in Fig. (4). As predicted earlier, the nonlinearity coefficient observed for $\mathrm{CCl}_{4} \mathrm{LCPCF}$ $\left(\gamma=0.0937 W^{-1} m^{-1}\right)$ has increased to $0.119 W^{-1} m^{-1}$ for the case of $\mathrm{S}_{-\mathrm{CCl}} \mathrm{LCPCF}$ (refer Tab.II). From the above understanding, its quite obvious that the nonlinearity associated with the MOF structure can be significantly increased by infiltration of nonlinear liquids and further enhancement can be made possible by suspending the fiber core. With this conclusive evidence on the effect of $\mathrm{S}_{-} \mathrm{CCl}_{4} \mathrm{LCPCF}$ in enhancing the nonlinearity of the system, we demonstrate the enhancement of nonlinear mechanism in $\mathrm{S}_{-} \mathrm{CCl}_{4} \mathrm{LCPCF}$ by considering the most popular nonlinear process namely, supercontinuum generation.

\section{SUPERCONTINUUM GENERATION}

The equation governing the propagation of an ultrashort optical pulse in MOFs is given by the generalized nonlinear Schrödinger equation (GNLSE) of the form as follows [6],

$$
\begin{gathered}
\frac{\partial U}{\partial z}+\sum_{n=2}^{4} \beta_{n} \frac{i^{n-1}}{n !} \frac{\partial^{n} U}{\partial t^{n}}+\frac{\alpha}{2} U=i \gamma\left(1+i \tau_{\text {shock }} \frac{\partial}{\partial t}\right) \\
\times\left(U(z, t) \int_{-\infty}^{\infty} R\left(t^{\prime}\right)\left|U\left(z, t-t^{\prime}\right)\right|^{2} d t^{\prime}\right)
\end{gathered}
$$

where $U(z, t)$ represents the pulse envelope, $z$ and $t$ are the longitudinal coordinate and time in the moving reference frame, respectively. The dispersion coefficient $\beta_{n}$ attributes to the Taylor expansion of the propagation constant around the center frequency $\omega_{0}$ and $n$ being the order of dispersion. $\gamma$ and $\alpha$ respectively amounts to the fiber nonlinearity and losses. The time derivative term on the right-hand side of Eq. (6) takes into account the dispersion of the nonlinearity, which is usually associated with the optical shock formation. The typical characteristic timescale of the $t_{\text {shock }}=1 /\left(\omega_{0}\right)$. $R(t)$ is the response function which takes into account both the instantaneous electronic response and the delayed Raman response. The functional form of $R(t)$ can be written as

$$
R(t)=\left(1-f_{R}\right) \delta(t)+f_{R} h_{R}(t)
$$

where $h_{R}$ is the retarded response function and $f_{R}$ amounts to fractional contribution to the delayed Raman response. $\delta(t)$ is the Dirac delta function. In the present context, we have considered silica and $\mathrm{CCl}_{4}$ as the choice of the core-material, and the corresponding appropriate functional forms of the retarded function are as follows:

1. Silica

$$
h_{R}=\frac{\tau_{1}^{2}+\tau_{2}^{2}}{\tau_{1} \tau_{2}^{2}} \sin \left(\frac{t}{\tau_{1}}\right) e^{-\frac{t}{\tau_{2}}} \Theta(t)
$$

with $f_{R}=0.18, \tau_{1}=0.012 \mathrm{ps}$, and $\tau_{2}=0.032 \mathrm{ps}[57$, 58 ]. $\Theta(t)$ is the heavyside step function.

2. $\mathrm{CCl}_{4}$

$$
\begin{array}{r}
h_{R}=A_{1} e^{-\frac{t}{\tau}} \\
\sin \left(\omega_{1} t\right)+A_{2} e^{-\frac{t}{\tau}} \sin \left(\omega_{2} t\right) \\
+A_{3} e^{-\frac{t}{\tau_{1}}}\left(1-e^{-\frac{t}{\tau_{2}}}\right) \Theta(t)
\end{array}
$$

with $f_{R}=0.12, A_{1}=0.0080, A_{2}=0.0092$, and $A_{3}=0.1418 . \quad \tau=0.89 \mathrm{ps}, \tau_{1}=0.241 \mathrm{ps}$ and $\tau_{2}=0.472$ ps. $\omega_{1}=42.1 \mathrm{THz}$, and $\omega_{2}=60.60 \mathrm{THz}[57,59,60]$.

To investigate the propagation dynamics and SCG, we numerically solved Eq. (6) using split-step Fourier method (SSFM) with hyperbolic sech profile of the form $U(0, t)=\sqrt{P} \operatorname{sech}\left(t / \tau_{0}\right)$ as the initial envelope of the pulse at $z=0[6,57]$. Numerical simulations are carried out for an input pulse of width $\tau_{0}=200$ fs at pump wavelength $\lambda_{p}=1550 \mathrm{~nm}$. The fiber parameters used for simulations are listed in Tab. II. 

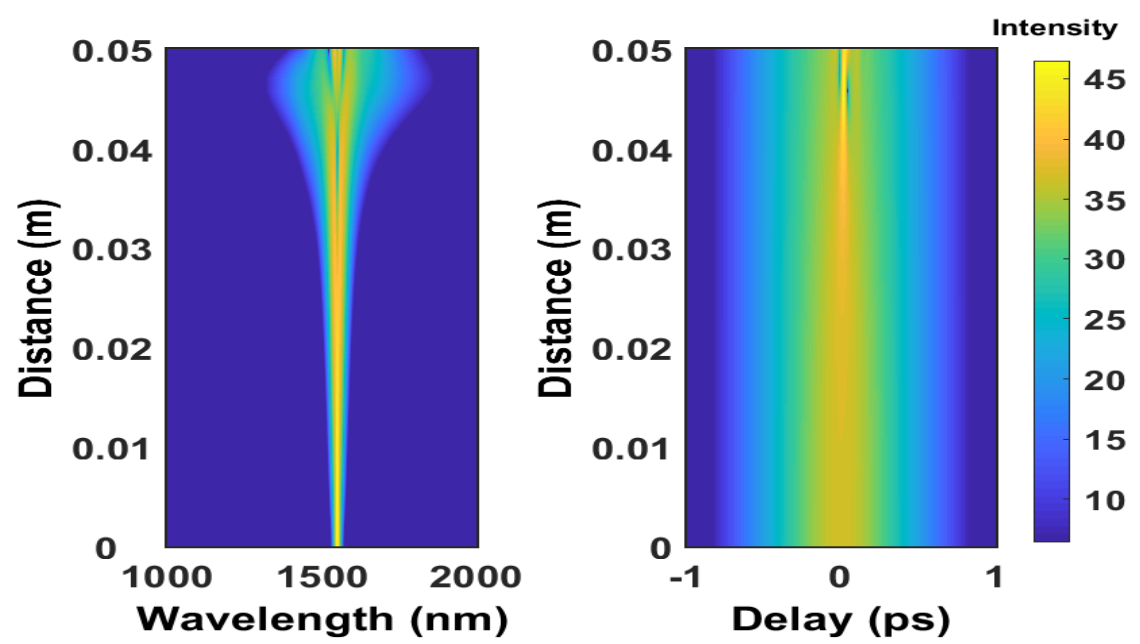

(a)
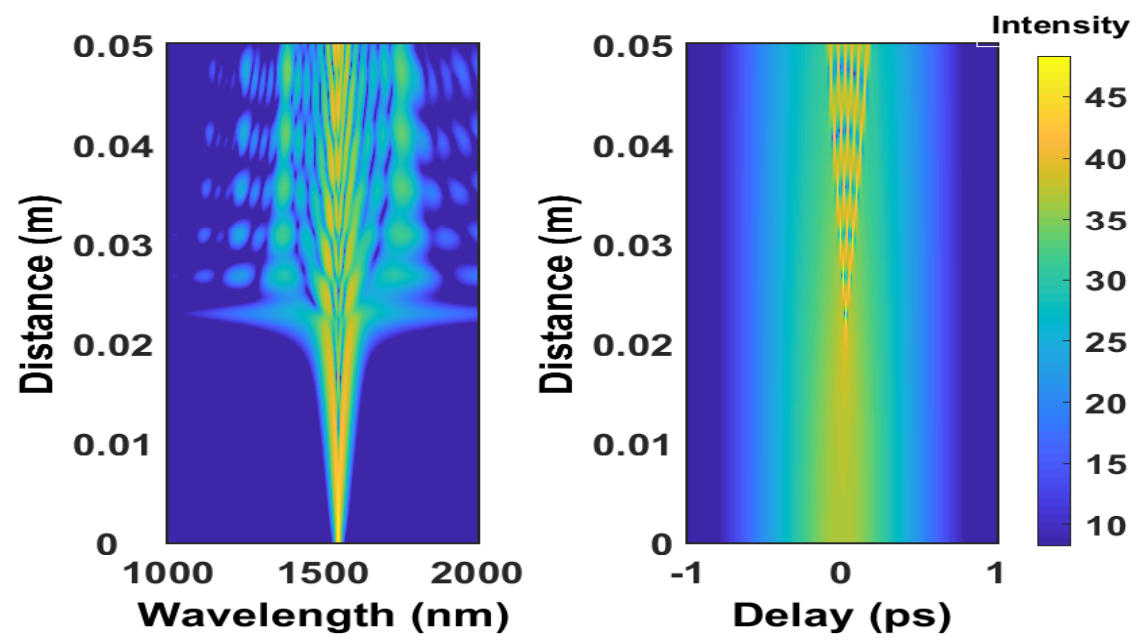

(b)

FIG. 5: (Color online) SC produced by an input pulse of power $5 \mathrm{~kW}$ in a MOF of length $\mathrm{L}=0.05 \mathrm{~m}$ for (a) Silica $\mathrm{PCF}$ (silica based solid core PCF), and (b) $\mathrm{CCl}_{4} \mathrm{LCPCF}\left(\mathrm{CCl}_{4}\right.$ infiltrated liquid core PCF).
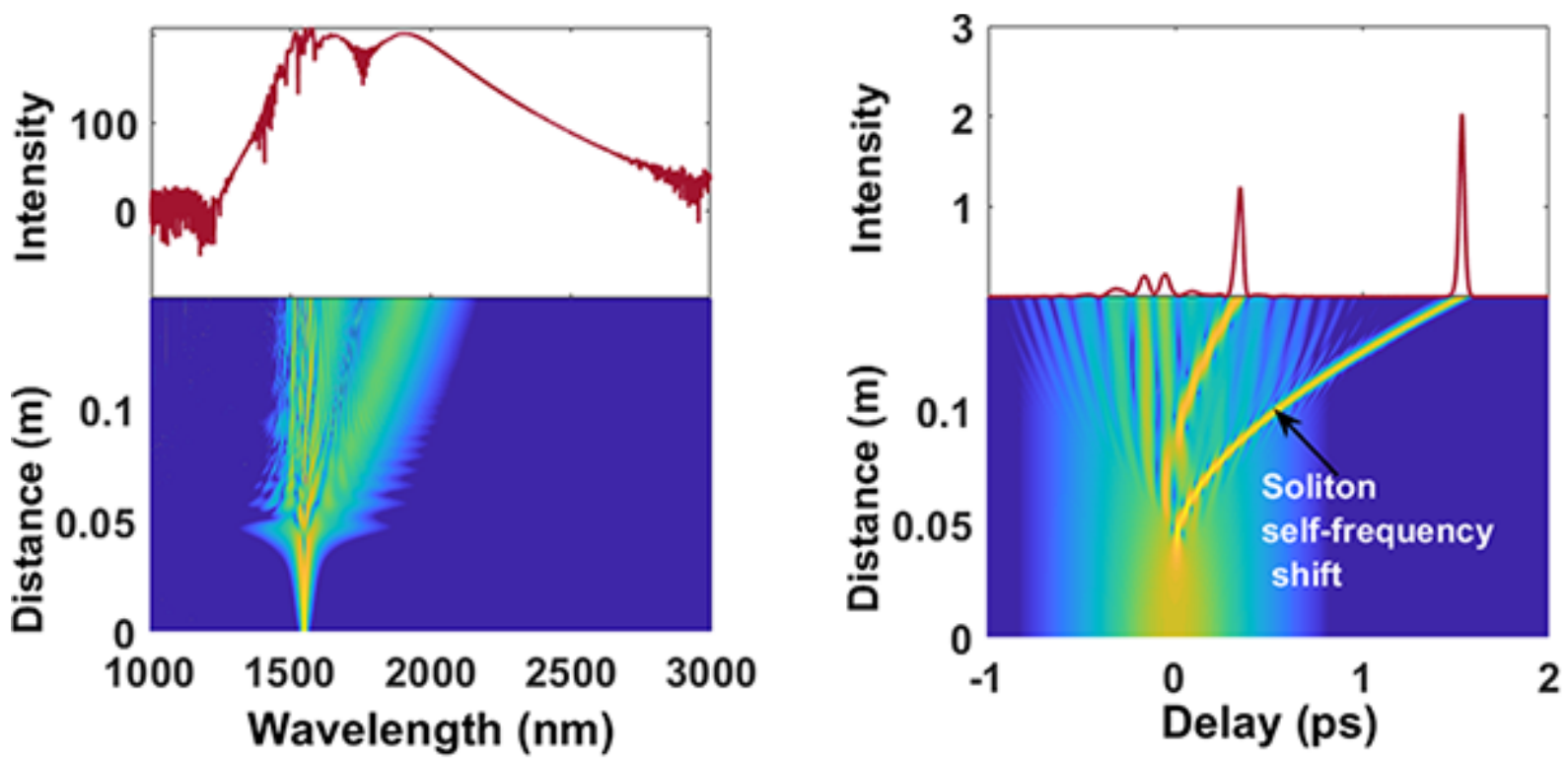

FIG. 6: (Color online) SCG through soliton fission mechanism by proposed silica PCF of length $0.15 \mathrm{~m}$ with $\mathrm{P}=5 \mathrm{~kW}$ and $\mathrm{T}_{0}=200 \mathrm{fs}$. 
TABLE III: Spectral characteristics of SC generated in different MOFs proposed with $\mathrm{P}=5 \mathrm{~kW}$ and $\mathrm{L}=0.05 \mathrm{~m}$ (Values are extracted from Fig. (5))

\begin{tabular}{|l|l|l|l|}
\hline MOFs & $\lambda_{\min }(n m)$ & $\lambda_{\max }(n m)$ & $\lambda_{\max }-\lambda_{\min }$ \\
\hline Silica PCF & 1364 & 1881 & 517 \\
\hline $\mathbf{C C l}_{4}$ LCPCF & 1300 & 2022 & 722 \\
\hline
\end{tabular}

followed by the influence of the core suspension in the broadband spectrum. To be more realistic in our numerical approach, we effectively included the fiber loss in the propagation dynamics. The effective loss $(\alpha)$ in the system is attributed to the sum of material absorption $\left(\alpha_{m}\right)$ and confinement/leakage $\left(\alpha_{c}\right)$ loss given by $\alpha=\alpha_{m}+\alpha_{c}$. Former is intrinsic to the nature of the material, while the latter depends on the fiber geometry and the structural parameters. We estimated the material absorption from the imaginary part of refractive index and can take the values $0.0002 \mathrm{~dB} / \mathrm{m}$, and $0.237 \mathrm{~dB} / \mathrm{m}$ respectively for silica and $\mathrm{CCl}_{4}$ [52]. The confinement loss is calculated from the imaginary part of the effective index using the expression $\alpha_{c}=8.67 k_{0} \operatorname{Im}\left\{\mathrm{n}_{\text {eff }}(\lambda)\right\}$ with $k_{0}=2 \pi / \lambda$ being the free space wavenumber. For all the fiber design discussed in the context, the confinement loss corresponding to the excitation wavelength $\left(\lambda_{p}=1550 \mathrm{~nm}\right)$ is negligibly small.

An input pulse with peak power $5 \mathrm{~kW}$ at excitation wavelength $1550 \mathrm{~nm}$ is coupled into PCF of length $0.05 \mathrm{~m}$ with different core materials considered. Fig. (5) depicts the evolutionary dynamics and the associated spectral broadening of conventional silica $\mathrm{PCF}$ and $\mathrm{CCl}_{4}$ infiltrated LCPCF. The underlying physical mechanism of supercontinuum generation is generally well known, typically, modulational instability and soliton fission are the two prominent mechanisms of broadband generation. Particularly, in the anomalous dispersion regime pumping, the spectral broadening leading to supercontinuum is typically governed by the soliton-driven dynamics. In the parametric space of our choice, the so-called soliton fission (SF) being the most important stage in the spectral broadening process, such that, the initial pulse profile set by the system parameters constitute a higher-order soliton which on perturbation breaks up into $\mathrm{N}$ soliton-like pulses. Depending on the combination of system parameters, the initial input profile changes, which substantially influence the spectral and temporal evolution which can be obvious from the subsequent discussion.

In order to highlight the physical process involved in the SCG, as a representative case we show in the Fig. (6), the evolutionary dynamics of the SCG in silica PCF for a fiber of length $0.15 \mathrm{~m}$ with same set of parameters as in Fig. (5). In similar lines to the earlier reports on SCG, it is apparent from the Fig. (6) that the initial stage of propagation is governed by a symmetrical spectral broadening $(<0.05 \mathrm{~m})$, resulting from the temporal compression of the propagating higher-order soliton. On further propagation, the perturbation resulting from higher order dispersion brings the onset of the soliton fission. The distance of the onset of the soliton fission is characteristic of the system which is set by its parameters. In principle, the injected higher-order soliton tends to split when its bandwidth reaches a maximum. In the present parametric space, both the higher order dispersion and Raman scattering accounts to perturbation and thereby contribute to fission of solitons. The constituent soliton resulting from the fission experiences a continuous shift due to soliton self-frequency shift resulting from Raman scattering. Arguably, it is apparent that the continuum generated by $\mathrm{CCl}_{4} \mathrm{LCPCF}$ is significantly broader than that of silica PCF. This can be explained in terms of soliton order $N$ defined as $\mathrm{N}=\mathrm{T}_{0} \sqrt{\frac{\gamma P}{\beta_{2}}}$. As the soliton order is proportional to the nonlinearity coefficient, one can effectively increase the soliton order by infiltrating the LCPCF with highly nonlinear liquids. For the proposed MOFs, $N \approx 7$ and $N \approx 16$ is observed respectively for silica $\mathrm{PCF}$ and $\mathrm{CCl}_{4} \mathrm{LCPCF}$. It can also be noticed that the soliton order also depends on dispersion coefficient as $\mathrm{N} \propto 1 / \beta_{2}$, and in comparison with silica $\mathrm{PCF}, \mathrm{CCl}_{4}$ infiltrated LCPCF shows relatively lower dispersion coefficient and thus contribute to increase in the soliton order $(N)$. Increased soliton order of the pulse used in $\mathrm{CCl}_{4}$ LCPCF through nonlinearity and dispersion management result in effective spectral broadening in a short length of fiber as evident from Fig. (5b). Also from the theory of soliton fission, the soliton pulse having shorter pulse width tend to suffer a large self frequency shift given by the expression $\mathrm{d} \gamma_{R} / \mathrm{dz} \propto \beta_{2} / \mathrm{T}_{0}^{4}$ [61]. The estimated pulse width of the shortest soliton after fission by using the formula $T_{j}=T_{0} /(2 \mathrm{~N}-2 \mathrm{j}+1)[62]$ with $\mathrm{j}=1$ ( for first ejected soliton) is found to be 16 fs and $6.3 \mathrm{fs}$ for the case of silica PCF and $\mathrm{CCl}_{4}$ infiltrated LCPCF. This shorter width of the first ejected soliton in the case of LCPCF contribute significantly to the broadening of the spectrum. In addition to the spectral extension on the long wavelength side, a narrow band resonance on the normal group velocity dispersion regime can be noticed, which is identified as the dispersive wave generation. A broad continuum spanning $\approx 722 \mathrm{~nm}$ is generated by using $\mathrm{CCl}_{4} \mathrm{LCPCF}$ as evident from Fig. (5b). A reference corresponding to an intensity level of 20 in arbitrary units is chosen to estimate the bandwidth of the spectrum. Highly broad continuum generation in $\mathrm{CCl}_{4} \mathrm{LCPCF}$ is attributed to the high nonlinearity coefficient provided by the $\mathrm{CCl}_{4}$ liquid along with the reduced dispersion parameter. Tab. III display the estimated spectral bandwidth at a reference level of 20 (a.u).

Having acquired a comprehensive understanding of the influence of liquid infiltration in the enhancement of spectral broadening process, in what follows, we describe the effect of core suspension in SCG. To highlight core suspension, we consider silica $\mathrm{PCF}$ and $\mathrm{CCl}_{4} \mathrm{LCPCF}$ as two representative fiber of choice for our investigation. It is apparent from section II, that the suspension of the core enhances the nonlinearity of the fiber. For numerical appreciation, the nonlinearity coefficient of the $\mathrm{S}_{-} \mathrm{CCl}_{4}$ LCPCF is $\approx 1.3$ times higher than the $\mathrm{CCl}_{4} \mathrm{LCPCF}$ without suspension effect. Thus one can expect that this increase in nonlinearity to further promote the spectral broadening process. As it is obvious from Fig. (7) portraying the spectral evolution of silica and $\mathrm{CCl}_{4}$ infiltrated suspended core MOFs, the combination of liquid infiltration and suspension dramatically enhance the 


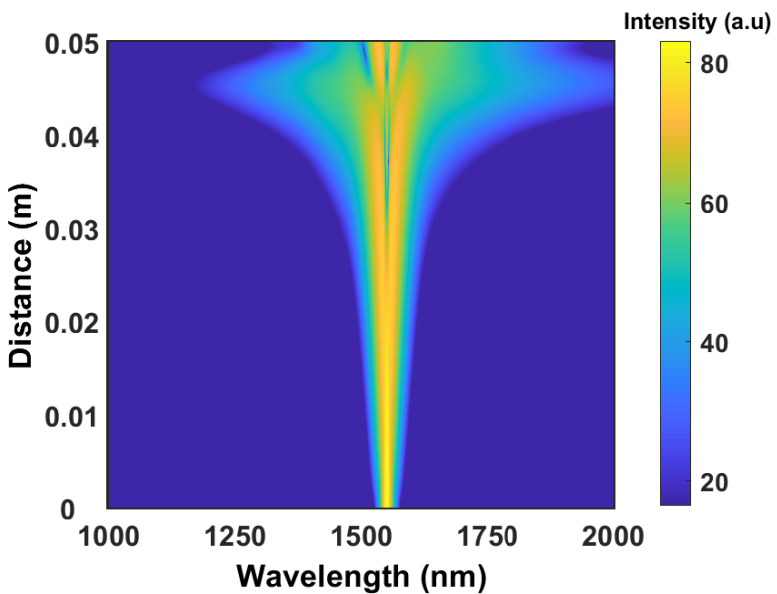

(a)

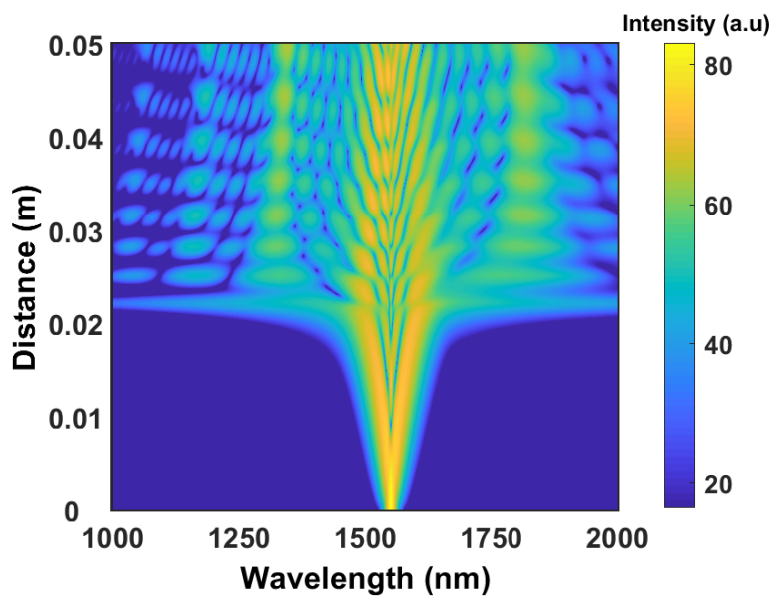

(b)

FIG. 7: (Color online) SC produced in (a) S-silica PCF (silica based suspended core PCF), and (b) S-CCl $\mathrm{LCPCF}_{4}$ $\left(\mathrm{CCl}_{4}\right.$ infiltrated suspended $\mathrm{LCPCF}$ for an input pump power $\mathrm{P}=5 \mathrm{~kW}$.

TABLE IV: Spectral characteristics of SC generated in silica PCF and $\mathrm{CCl}_{4} \mathrm{LCPCF}$ with and without suspension effect with power $\mathrm{P}=5 \mathrm{~kW}$ at length $\mathrm{L}=0.05 \mathrm{~m}$ (Values are extracted from Fig. (8) at a reference intensity 20 [a.u]).

\begin{tabular}{|l|l|l|l|}
\hline $\begin{array}{l}\text { Micro structured optical fiber } \\
\text { type }\end{array}$ & $\begin{array}{l}\lambda_{\min } \\
(\mathrm{nm})\end{array}$ & $\begin{array}{l}\lambda_{\max } \\
(\mathrm{nm})\end{array}$ & $\begin{array}{l}\lambda_{\max }-\lambda_{\min } \\
(\mathrm{nm})\end{array}$ \\
\hline Silica PCF $/$ S-Silica PCF & $1364 / 1366$ & $1881 / 1933$ & $517 / 567$ \\
\hline $\mathbf{C C l}_{4} \mathbf{L C P C F}_{\mathbf{S}-\mathbf{C C l}_{4} \mathbf{L C P C F}}$ & $1300 / 1045$ & $2022 / 2298$ & $722 / 1253$ \\
\hline
\end{tabular}

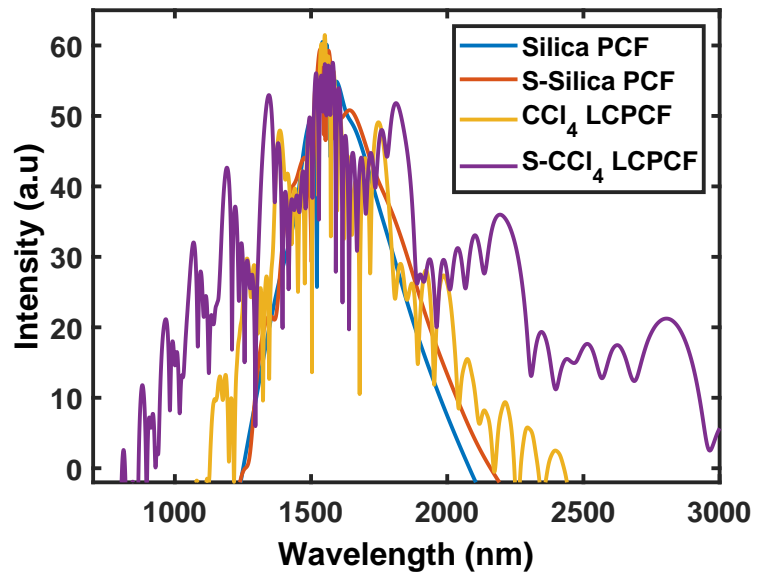

FIG. 8: (Color online) Spectral output with and without suspension for silica $\mathrm{PCF}$ and $\mathrm{CCl}_{4}$ infiltrated $\mathrm{LCPCF}$ for an input power of $\mathrm{P}=5 \mathrm{~kW}$ at $\mathrm{L}=0.05 \mathrm{~m}$.

spectral broadening process.

To emphasize the role of the core suspension in SCG, we show in Fig. (8) the spectral output in silica PCF and $\mathrm{CCl}_{4}$ infiltrated LCPCF with and without suspension for an input power of $5 \mathrm{~kW}$ in $0.05 \mathrm{~m}$ length of fiber. The recorded spectral width at a reference level of 20 (a.u) for conventional silica PCF and S-Silica PCF are $517 \mathrm{~nm}$ and $567 \mathrm{~nm}$, while, $\mathrm{CCl}_{4}$ infiltrated $\mathrm{LCPCF}$ register a bandwidth of $722 \mathrm{~nm}$ and $1253 \mathrm{~nm}$, respectively for LCPCF with and without suspension. The results of the simulations are outlined in Tab. IV. The substantial

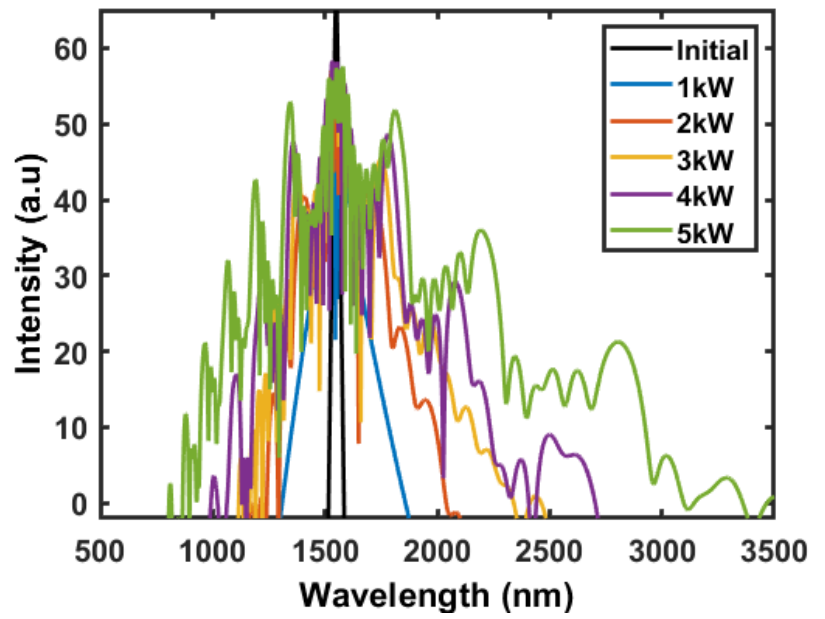

FIG. 9: (Color online) Variation of SC spectra for different input power at $\mathrm{L}=0.05 \mathrm{~m}$ with the proposed S-CCl ${ }_{4} \mathrm{LCPCF}$ (suspended $\mathrm{CCl}_{4}$ infiltrated liquid core photonic crystal fiber).

enhancement in the spectral width is due to the fact that, the suspended core increases the nonlinearity coefficient further along with decrease in dispersion coefficient. This results in increasing the soliton order from $\mathrm{N}=6.7$ (16.3) for the case of silica PCF $\left(\mathrm{CCl}_{4}\right.$ $\mathrm{LCPCF}$ ) to $\mathrm{N}=8.7$ (20.7) corresponding to S-Silica PCF $\left(\mathrm{S}-\mathrm{CCl}_{4} \mathrm{LCPCF}\right)$. This increase in soliton order not only increase the number of constituent solitons derived from fission, but also significantly reduce the width of the ejected soliton which consequently enhances the spectral 
TABLE V: Spectral characteristics of SC generated in $\mathrm{S}_{-} \mathrm{CCl}_{4} \mathrm{LCPCF}$ with different input powers (for a fiber length $0.05 \mathrm{~m}$ ) and propagation length (with input peak power $5 \mathrm{~kW}$ )(Values are extracted from Fig. (9) and

Fig. (10)) at a reference intensity 20 (a. u).

\begin{tabular}{|c|l|l|c|}
\hline Power $(\mathbf{k W})$ & $\lambda_{\min }(\mathrm{nm})$ & $\lambda_{\max }(\mathrm{nm})$ & $\lambda_{\max }-\lambda_{\min }$ \\
\hline 1 & 1395 & 1805 & 410 \\
\hline 2 & 1324 & 1909 & 585 \\
\hline 3 & 1258 & 2015 & 757 \\
\hline 4 & 1159 & 2138 & 979 \\
\hline 5 & 1045 & 2298 & 1253 \\
\hline Length (m) & $\lambda_{\min }(\mathrm{nm})$ & $\lambda_{\max }(\mathrm{nm})$ & $\lambda_{\max }-\lambda_{\min }$ \\
\hline 0.01 & 1479 & 1624 & 145 \\
\hline 0.02 & 1315 & 1786 & 471 \\
\hline 0.03 & 1178 & 2009 & 831 \\
\hline 0.04 & 1089 & 2228 & 1139 \\
\hline 0.05 & 1045 & 2298 & 1253 \\
\hline
\end{tabular}

broadening.

For better insight, we study the effect of input power and fiber length on the spectral broadening process. Firstly, we study the impact of pump power at fixed length of fiber. Fig. (9) depicts the spectral evolution of $\mathrm{S}-\mathrm{CCl}_{4} \mathrm{LCPCF}$ of length $\mathrm{L}=0.05 \mathrm{~m}$ for a range of pump powers. In given parametric space of choice, any increase in the pump power invariably broadens the continuum as shown in the Fig. (9). This is attributed to the fact that the increasing pump power tends to monotonously increase the effective nonlinear contribution and hence broadens the spectrum. The effect of fiber length on SCG is studied by passing an input pulse of power $5 \mathrm{~kW}$ through fibers for some representative length and the corresponding spectral output is shown in Fig. (10). Similar to the effect of power, the continuum generated in $\mathrm{CCl}_{4}$ S-LCPCF depends on the length of the fiber segment, such that, the width of continuum generated increases monotonically with length. An overview of the results obtained are tabulated in Tab.V. It is very obvious to understand that the $\mathrm{CCl}_{4}$ infiltrated LCPCF structures are superior in terms of the nonlinearity and thus inherently enhances spectral broadening. Also, the proposed new design with suspended core further elevates the nonlinearity, thus enabling one to achieve broad spectrum at the shortest possible distance than the rest of the combinations of fiber design so far realized.

\section{CONCLUSION}

In summary, a highly nonlinear suspended liquid core photonic crystal fiber has been proposed for different nonlinear applications. The optical characteristics such as dispersion, nonlinearity have been studied and compared with conventional silica counterpart. An ultra broad supercontinuum of width $\approx 722 \mathrm{~nm}$ was simulated by passing a $5 \mathrm{~kW}$ soliton-like pulse of width $0.2 \mathrm{ps}$ through a $\mathrm{CCl}_{4}$ filled liquid core photonic crystal fiber of length $0.05 \mathrm{~m}$. The broad continuum generation in $\mathrm{CCl}_{4}$
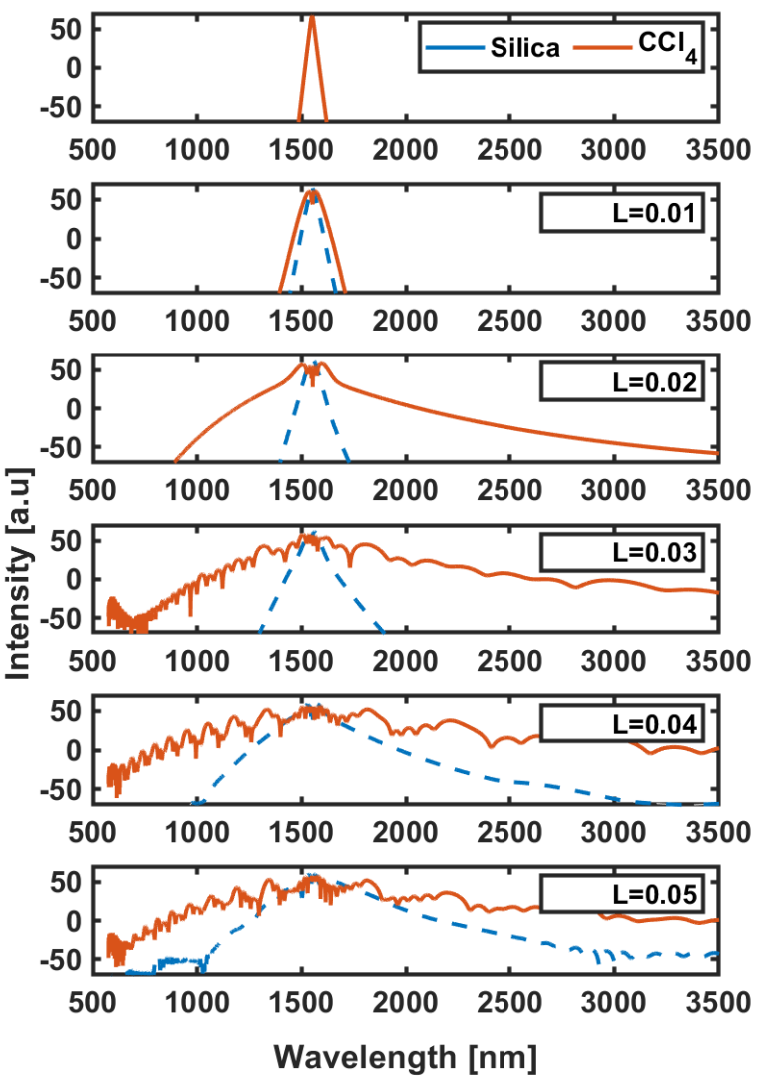

FIG. 10: (Color online) Variation of SC spectra for different lengths of propagation for an input power $\mathrm{P}=5 \mathrm{~kW}$ with the proposed $\mathrm{S}-\mathrm{CCl}_{4} \mathrm{LCPCF}$ and S-Silica $\mathrm{PCF}$.

$\mathrm{LCPCF}$ is attributed to the high nonlinearity resulting from large nonlinear refractive index. In order to further enhance the supercontinuum generation, suspended core MOFs with suspension factor (SF) of 1.27 was considered. An enhancement in $\mathrm{SC}$ width from $722 \mathrm{~nm}$ to $1253 \mathrm{~nm}$ has been observed in $\mathrm{S}-\mathrm{CCl}_{4} \mathrm{LCPCF}$ in the similar simulation condition. The variation of continuum width with input pulse power and propagation distance are also studied. Although we limit the present study to $\mathrm{CCl}_{4}$, the aforementioned results are universal and can be adopted to any different material medium. To conclude, we successfully explained a new means to enhance the nonlinearity of the microstructure waveguides, which could potentially find new applications in photonic technologies. Considering the recent technological development of supercontinuum based laser source, we believe the proposed suspended liquid core photonic crystal fiber can be a new class of fibers for next generation of broadband sources. 
Atkin, "All-Silica Single-Mode Optical Fiber with Photonic Crystal cladding", Optics Letters, 21, 1547 (1996).

[3] R. F. Cregan, B. J. Mangan, J. C. Knight, T. A. Birks, P. S. J. Russell, P. J. Roberts and D. C. Allan, "Single mode photonic band gap guidance of light in air", Science, 285, 1537 (1999).

[4] J. C. Knight, J. Broeng, T. A. Birks and P. St. J. Russell, "Photonic Band Gap Guidance in Optical Fibers", Science, 282, 1476 (1998).

[5] R.V.J. Raja, K. Senthilnathan, K. Porsezian and K. Nakkeeran, "Efficient Pulse Compression Using Tapered Photonic Crystal Fiber at $850 \mathrm{~nm}$ ", J. Quantum Electron., 46, 12 (2010).

[6] J. M. Dudley and S. Coen, "Supercontinuum generation in photonic crystal fiber", Rev. Mod. Phys., 78(4), 1135 (2006).

[7] J. M. Dudley, and J.R Taylor, "Supercontinuum generation in optical fibers", Cambridege University Press (2010).

[8] M. Chen, S.G. Yang, , F. F. Yin, H. W. Chen and S. Z. Xie, "Design of a New Type High Birefringence Photonic Crystal Fiber", Optoelectron. Lett., 4, 19 (2008).

[9] T. A. Birks, J. C. Knight and P. St. J.Russell, "Endlessly Single-Mode Photonic Crystal Fiber", Opt. Lett., 22, 961 (1997).

[10] K. M. Ho, C. T. Chan and C. M. Soukoulis, "Existence of a Photonic Gap in Periodic Dielectric Structures", Phys. Rev. Lett., 65, 3152 (1990).

[11] J. D. Joannopoulos, S. G. Johnson, J. N. Winn and R. D. Meade, "Photonic Crystals: Molding the Flow of Light", Princeton University Press (2011).

[12] F. Poli, A. Cucinotta, M. Fuochi, S. Selleri and L. Vincetti, "Characterization of micro structured optical fibers for wideband dispersion compensation", J. Opt. Soc. Am. A, 20, 1958 (2003).

[13] F. Poli, A. Cucinotta, S. Selleri and A. H. Bouk, "Tailoring of flattened dispersion in highly nonlinear photonic crystal fibers", Photon. Technol. Lett., 16, 1065 (2004).

[14] S. E. Kim, B. H. Kim, C. G. Lee, S. Lee, K. Oh and Chul-Sik Kee, "Elliptical defected core photonic crystal fiber with high birefringence and negative flattened dispersion", Opt. Express, 20, 1385 (2012).

[15] K. Saitoh and M. Koshiba, "Chromatic dispersion control in photonic crystal fibers: application to ultra-flattened dispersion", Opt. Express, 11, 843 (2003).

[16] Bo-Wen Liu, Ming-Lie Hu, Xiao-Hui Fang, Yan-Feng Li, L. Chai, Ching-Yue Wang, W. Tong, J. Luo, A. A. Voronin and A. M. Zheltikov, "Stabilized soliton selffrequency shift and $0.1-\mathrm{PHz}$ sideband generation in a photonic-crystal fiber with an air-hole-modified core", Opt. Express, 16, 14987 (2008).

[17] B. Kibler, P. A. Lacourt, F. Courvoisier, and J. M. Dudley, "Soliton spectral tunnelling in photonic crystal fibre with subwavelength core defect", Electron. Lett., 43, 967 (2007).

[18] E. E. Serebryannikov and A. M. Zheltikov, "Nanomanagement of dispersion, nonlinearity, and gain of photoniccrystal fibers: qualitative arguments of the Gaussianmode theory and nonperturbative numerical analysis", J. Opt. Soc. Am. B, 23, 1700 (2006).

[19] A. Sharafali, K. Nithyanandan and K. Porsezian, "Selfsimilar pulse compression by defective core photonic crystal fiber with cubic-quintic nonlinearities", Optik, 178, 591 (2019).

[20] J. H. V. Price, X. Feng, A. M. Heidt, G. Brambilla, P. Horak, F. Poletti, G. Ponzo, P. Petropoulos, M. Petrovich, J. Shi, M. Ibsen, W. H. Loh, H. N. Rutt, D. J. Richardson, "Supercontinuum generation in non-silica fibers", Opt. Fib. Techn., 18, 327 (2012).

[21] Y. Wang, M. Yang, D. N. Wang and C. R. Liao, "Selectively infiltrated photonic crystal fiber with ultra- high temperature sensitivity", Photon. Technol. Lett., 23, 1520 (2011).

[22] X. Zheng, Y. Liu, Z. Wang, T. Han, C. Wei and J. Chen, "Transmission and temperature sensing charecteristics of a selectively liquid-filled photonic band gap fiber", Appl. Phys. Lett., 100, 141104 (2012).

[23] Y. Huang, Y. Xu and A. Yariv, "Fabrication of functional microstructured optical fibers through a slective-filling technique", Appl. Phys. Lett., 85,5182 (2004).

[24] R. Spittel, D. Hoh, S. Bruckner, A. Schwuchow, K. Schuster, J. Kobelke and H. Bartelt, "Selective filling of metals into photonic crystal fibers", Proc. SPIE 7946, Photon. Phononic Propert. Eng. Nanostruct. 7946, 794602 (2011).

[25] A.Sharafali, K.Nithyanandan"Ultra-broadband continuum generation in silica based defective core photonic crystal fiber" Optik, 191,121-131 (2019).

[26] K Porsezian, K Nithyanandan, RVJ Raja, R Ganapathy "A theoretical investigation of soliton induced supercontinuum generation in liquid core photonic crystal fiber and dual core optical fiber", The European Physical Journal Special Topics 222 (3-4), 625-640 (2013).

[27] M. Chemnitz, M. Gebhardt, C. Gaida, F. Stutzki, J. Kobelke, J. Limpert, "Hybrid soliton dynamics in liquidcore fibres", Nature Comm., 8, 1 (2017).

[28] C. P. Yu and J. H. Liou, "Selectively liquid-filled photonic crystal fibers for optical devices", Opt. Express, 17, 8729 (2009).

[29] C. P. Yu, J. H. Liou, S. S. Huang and H. C. Chang, "Tunable dual-core liquid-filled photonic crystal fibers for dispersion compensation", Opt. Express, 16, 4443 (2008).

[30] M. Cordier, A. Orieux, R. Gabet, T. Harle, N. Dubreuil, E. Diamanti, P. Delaye and I. Zaquine, "Liquid filled photonic crystal fiber: A flexible tool for fibered photon-pair generation", QIM, QW3C.5 (2017).

[31] S. K. Methaprian, N. Ayyanar, P. Mahalakshmi, M. Sumathi, D. Vigneswaran and M. S. M. Rajan, "Design of temperature sensor using liquid filled photonic crystal fiber", IEEE-CRALT, pp.1-5 (2016).

[32] Y. E. Monfared and S. A. Ponomarenko, "All-optical wavelength conversion using a liquid-filled photonic crystal fiber", Photonics North (PN),pp.1-1 (2017).

[33] D. Ghosh, S. Bose, S. Roy and S. K. Bhadra, "Design and fabrication of microstructured optical fibers With optimized core suspension for enhanced supercontinuum generation", J. Lightwave Technol., 33, 4156 (2015).

[34] J. K. Ranka, R. S. Windeler and A. J. Stentz, "Visible continuum generation in air-silica microstructure optical fibers with anomalous dispersion at $800 \mathrm{~nm}$ ", Opt. Lett., 25,25 (2000).

[35] A M Zheltikov, "Let there be white light: supercontinuum generation by ultrashort laser pulses", PhysicsUspekhi, 49, 605 (2006).

[36] K. Nithyanandan, R. Vasantha Jayakantha Raja, K. Porsezian and T. Uthayakumar, "A colloquium on the influence of versatile class of saturable nonlinear responses in the instability induced supercontinuum generation", J. Opt. Fib. Tech., 19, 348 (2013).

[37] K. Nithyanandan, R. Vasantha Jayakantha Raja, and K. Porsezian, "Power play in the supercontinuum spectra of saturable nonlinear media", Laser Phys. 24045405 (2014).

[38] W. J. Wadsworth, A. Ortigosa-Blanch, J. C. Knight, T. A. Birks, T. P. M. Man and P. St. J. Russell, "Supercontinuum generation in photonic crystal fibers and optical fiber tapers: a novel light source", J. Opt. Soc. Am. B, 19, 2148 (2002).

[39] G. Genty, S. Coen, and J. M. Dudley, "Fiber supercontinuum sources (Invited)", J. Opt. Soc. Am. B, 24, 1771 (2007).

[40] J. M. Dudley and J. R. Taylor, "Ten years of nonlinear optics in photonic crystal fibre", Nat. Photonics, 3, 85 
(2009).

[41] M. Nisoli, S. D. Silvetri, O. Svelto, R. Szipocs, K. Ferencz, C. Spielmann,S. Sartania and F. Krausz, "Compression of high-energy laser pulses below $5 \mathrm{fs",} \mathrm{Opt.}$ Lett., 22, 522 (1997).

[42] T. Udem, R. Holzwarth and T. W. Hansch, "Optical frequency metrology", Nature, 416, 233 (2002).

[43] I. Hartl, X. D. Li, C. Chudoba, R. K. Ghanta, T. H. Ko, J.G. Fujimoto, J. K. Ranka and R. S. Windeler, "Ultrahigh-resolution optical coherence tomography using continuum generation in an air silica microstructure optical fiber", Opt. Lett., 26, 608 (2001).

[44] R. R. Alfano, "The Supercontinuum Laser SourceFundamental with Updated References", 2nd ed. New York: Springer(2006).

[45] A. Demircan and U. Bandelow, "Analysis of the interplay between soliton fission and modulation instability in supercontinuum generation", Appl. Phys. B, 86, 31 (2007).

[46] F. Li, Q. Li, J. Yuan and P. K. A. Wai, "Highly coherent supercontinuum generation with picosecond pulses by using self-similar compression", Optic exp., 22, 27339 (2014).

[47] T. J. Bridges, A. R. Chraplyvy, J. G. Bergman and R. M. Hart, "Broadband infrared generation in liquid-brominecore optical fibers", Opt. Lett., 7, 566 (1982).

[48] R. Zhang, J. Teipel and H. Giessen, "Theoretical design of a liquid-core photonic crystal fiber for supercontinuum generation", Opt. Exp., 14, 6800 (2006).

[49] M. Vieweg, S. Pricking, T. Gissibl, Y. V. Kartashov, L. Torner and H. Giessen, "Tunable ultrafast nonlinear optofluidic coupler", EPJ Web of Conferences, 41, 12010 (2013).

[50] S. Pricking and H. Giessen, "Generalized retarded response of nonlinear media and its influence on soliton dynamics", Opt.Exp., 19, 2895 (2011).

[51] S. Guo, F. Wu, S. Albin, H. Tai and R. S. Rogowski, "Loss and dispersion analysis of microstructured fibers by finite-difference method", Opt. Exp., 12, 3341 (2004)

[52] S. Kedenburg, M. Vieweg, T. Gissibl and H. Giessen, "Linear refractive index and absorption measurements of nonlinear optical liquids in the visible and near-infrared spectral region", Opt. Mat. Exp., 2, 1588 (2012).

[53] G. Ghosh, M. Endo and T. Iwasaki, "Temperaturedependent Sellmeier coefficients and chromatic dispersions for some optical fiber glasses", J. Lightwave Technol., 12, 1338 (1994).

[54] G. Farin, "Curves and Surfaces for CAGD",5th edition, Academic Press (2001).

[55] J. M. Dudley, C. Flinot, D. J. Richardson and G. Milot, "Self-similarity in ultrafast nonlinear optics", Nat. Phys., 3, 597 (2007).

[56] S. Couris, M. Renard, O. Faucher, B. Lavorel, R. Chaux, E. Koudoumas and X. Michaut, "An experimental investigation of the nonlinear refractiveindex $\left(n_{2}\right)$ of carbon disulfide and toluene by spectral shearing interferometry and z-scan techniques", Chem. Phys. Lett., 369, 318 (2003).

[57] G. P. Agrawal, "Nonlinear Fiber Optics", 5th edition, New York: Academic press (2013).

[58] R. H. Stolen, J. P. Gordon, W. J. Tomlinson, and H. A. Haus, "Raman response function of silica-core fibers", 6 , 1159 (1989).

[59] M. Chemnitz, C. Gaida, M. Gebhardt, F. Stutzki, J. Kobelke, A. Tünermann, J. Limpert and M. A. Schmidt "Carbon chloride-core fibers for soliton mediated supercontinuum generation", 26, 3221 (2018).

[60] K. Itoh, Y. Toda, R, Moria, and M. Yamashita, "Coherent Optical Control of Molecular Motion Using Polarized Sequential Pulses", Jpn. J. Appl. Phys., 43, 6448 (2004).

[61] J. P. Gordon, "Theory of the soliton self-frequency shift", Opt. Lett., 11, 662 (1986).

[62] Y. Kodama and A. Hasegawa, "Nonlinear pulse propagation in a monomode dielectric guide", IEEE Photonics Technol. Lett., QE-23, 510 (1987). 